\title{
COMPRIMENTO TOTAL DO INTESTINO EM BOVINOS DE ORIGEM EUROPEIA
}

\author{
Orlando M. PAIVA * \\ Vicente BORELLI ** \\ José PEDUTI NETO ***
}

\begin{abstract}
RFMV-A/21
PAIVA, O.M.; BORELLI, V.; PEDUTI NETO, J. Comprimento total do intestino em bovinos de origem Européia. Rev. Fac. Med. vet. Zootec. Univ. S. Paulo, 14(2): 189-197, 1977.

RESUMO: Foi estudado o comprimento dos vários tratos do intestino, bem como seu comprimento total, em bovinos de raças européias, 20 machos e 20 fêmeas, adultos e encontradas iseguintes medidas:
\end{abstract}

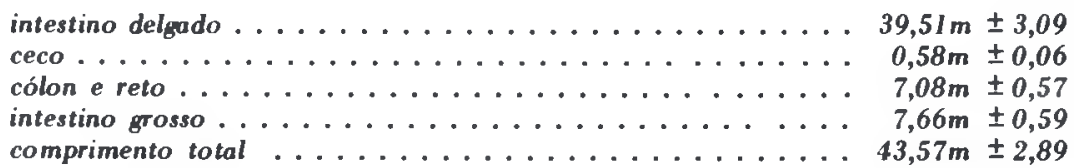

A análise estatistica não mostrou diferenças significantes entre os sexos, nem correlação entre o comprimento total do intestino com o peso ou o comprimento da carcaça.

UNITERMOS: Anatomia, bovinos *; Intestinos, anatomia *.

\section{INTRODUÇĀO E LITERATURA}

Tendo PAIVA \& BORELLI (1962) determinado, em recente pesquisa, as dimensões do trato entérico nos mestiços de zebu, procuramos agora, perfilhando igual metodologia, conhecer, em bovinos de raças européias, o comprimento do intestino delgado, o do ceco, o do cólon e reto conjuntamente, enfim de todo o tubo intestinal, vi- sando, não só confronto entre estes dados e os obtidos por aqueles AA. em zebuinos, mas também, comparação com os ensinamentos exarados nos compêndios de , Anatomia Veterinária, cujas descriçôes esteiam-se, provavelmente, na morfologia de taurinos.

Assim, compulsando os tratados didáticos, colhemos, no tocante à extensão dos diferentes segmentos do intestino, as informações abaixo relacionadas em tabela:

- Trabalho comunicado ao X Congresso Brasileiro de Anatomia, realizado em São Paulo de 10 a 14 de julho de 1967. 


\begin{tabular}{|c|c|c|c|c|c|}
\hline AUTORES & ANO & $\begin{array}{c}\text { INTESTINO } \\
\text { DELGADO }\end{array}$ & $\begin{array}{l}\text { INTESTINO } \\
\text { GROSSO }\end{array}$ & CECO & TOTAL \\
\hline CARADONA \& BOSSI & s.d. & $40-45$ & - & 0,80 & - \\
\hline MONGIARDINO ${ }^{17}$ & 1905 & - & - & 0,80 & - \\
\hline MONTANE \& BOURDELLE 18 & 1917 & 40 & $7,70-8,75$ & $0,70-0,75$ & $\pm 47,70-48,75$ \\
\hline LEPOUTRE ${ }^{13}$ & 1921 & 40 & - & - & - \\
\hline$L E S B R E^{14}$ & 1922 & 40 & $8-10$ & - & - \\
\hline CARADONA ${ }^{4}$ & 1930 & $40-45$ & - & 0,80 & - \\
\hline FAVILLI ${ }^{7}$ & 1931 & 40 & $7,70-8,80$ & $0,70-0,80$ & $\pm 47,70-48,80$ \\
\hline ELLENBERGER \& BAUM & 1932 & $40-49$ & $6,40-11$ & - & $39-63$ \\
\hline MARTIN \& SCHAUDER ${ }^{15}$ & 1935 & $30-45$ & $6,40-10$ & - & $39-59$ \\
\hline SCHMALTZ $Z^{21}$ & 1938 & - & 8,20 & - & - \\
\hline$F A V I L L I^{8}$ & 1943 & 40 & $7-8$ & - & - \\
\hline$B R U N I \& Z I M M E R L^{2}$ & 1947 & $40-45$ & $8-10$ & - & - \\
\hline ZIMMERL ${ }^{24}$ & 1949 & 40 & $\pm 8,10-9,10$ & 0,50 & $\pm 48,10-49,10$ \\
\hline$K L I M O V^{12}$ & 1953 & - & $6,40-10$ & - & - \\
\hline DOBBERSTEIN \& KOCH ${ }^{5}$ & 1954 & - & - & - & $39-63$ \\
\hline$M A S S U I^{16}$ & 1960 & $40-49$ & $6,40-11$ & $0,50-0,60$ & $39-63$ \\
\hline NICKEL \& SCHUMMER ${ }^{21}$ & 1960 & $27-49$ & $6,50-14$ & $0,50-0,70$ & $33-63$ \\
\hline $\begin{array}{l}\text { GONZALES Y GARCIA \& } \\
\text { ALVAREZ }\end{array}$ & 1961 & 45 & $10-12$ & - & $55-57$ \\
\hline SCHWARZE \& SCHRODER ${ }^{22}$ & 1962 & $27-49$ & $6,50-14$ & - & $33,50-63$ \\
\hline$K A T O^{11}$ & 1963 & $\pm 4 I$ & \pm 10 & 0,75 & - \\
\hline SISSON \& GROSSMAN $N^{23}$ & 1965 & 40 & $\pm 11,05$ & 0,75 & $\pm 51,05$ \\
\hline
\end{tabular}

Já, em trabalho especializado, BLACK, SEMPLE e LUSH' (1934), buscando, entre mestiços meio sangue, Zebu-Hereford e Zebu-Shorthorn, e bovinos puro sangue Hereford e Shorthorn. possíveis diferenças no comprimento do intestino, concluem serem destituídas de significação estatística as divergências registradas, cabendo lembrar que, entretanto, os AA. trabalharam com animais jovens.

De outra parte, de GIROLA. $\mathrm{MO}^{9}(1950)$, em pesquisa voltada a estudos comparativos sobre a morfologia e o comprimento do intestino, entre Bos taurus e Bos bubalus, encontra para a primeira espécie, representada por 10 animais, 3 machos e 7 fêmeas, de um a sete anos, extensão total, na maior parte das vêzes, de 47 a $50 \mathrm{~m}$ para o tubo entérico; entretanto, fornece-nos ainda o autor, dados que permitem sejaın calculadas as dimensões médias dos diversos seg- mentos, bem como apontadas suas medidas máxima e mínima; assim, o intestino delgado oscilando de 35,50 a $42,00 \mathrm{~m}$, mostra como valor médio $39,42 \mathrm{~m}$; o ceco, variando de 0,50 a $0,80 \mathrm{~m}$, figura, em média, com $0,70 \mathrm{~m}$; o cólon e o reto, em conjunto, medindo de 6,03 a $7,95 \mathrm{~m}$, exibem o comprimento médio de $7,30 \mathrm{~m}$; por fim, a extensão total média do intestino, equivale a $47,52 \mathrm{~m}$, sendo a mínima $42,03 \mathrm{~m}$ e, a máxima $50,70 \mathrm{~m}$. Acrescenta também, o autor, ter assinalado correlação positiva das dimensões do órgão com a altura e com o comprimento do animal.

Finalmente, PAIVA e BOREL$L^{1}{ }^{19}(1962)$, visando determinar a extensão do trato entérico em mestip̧os de zebu, registram, em média, para o intestino delgado. $30,72 \pm 1,99 \mathrm{~m}$ nos machos e $31,58 \pm 3,24 \mathrm{~m}$, nas fêmeas, ou, média global de $31,15 \pm 2,73 \mathrm{~m}$, e valores máximo e mínimo de $40,07 \mathrm{~m}$ e $27,14 \mathrm{~m}$; para o ceco, encon- 
tram em média $0,51 \pm 0,12 \mathrm{~m}$ nos machos e $0,52 \pm 0,10 \mathrm{~m}$ nas fêmeas, vale dizer, média geral de $0,51 \pm 0,11 \mathrm{~m}$, consignando $0,94 \mathrm{~m} \mathrm{e}$ $0,35 \mathrm{~m}$, como limites; para o cólon e reto, assinalam, em média, $6,56 \pm 0,95 \mathrm{~m}$ nos machos e 7,03 $\pm 0,50 \mathrm{~m}$ nas fêmeas, com média global de $6,80 \pm 0,80 \mathrm{~m}$, compreendido entre os extremos de $9,67 \mathrm{~m}$ e $4,95 \mathrm{~m}$; por sua vez, para o intestino grosso, em conjunto, computam, em média $7,07 \pm 1,04 \mathrm{~m}$ nos machos e $7,55 \pm 0,56 \mathrm{~m}$ nas fêmeas, ou, média global de $7,31 \pm 0,75 \mathrm{~m}$, com medidas desde 10,6 ] até 5,44 ; finalmente, para todo o trato intes. tinal, apontam, em média, 37,78 $\pm 2,73$ nos machos e $39,13 \pm 3,64 \mathrm{~m}$ nas fêmeas, ou média global de $38,84 \pm 3,26 m$ com valores máximo de $48,03 \mathrm{~m}$ e mínimo de $33,62 \mathrm{~m}$.

Elucidam ainda, estes $\mathbf{A A}$, não terem encontrado diferenças significantes para os sexos, bem como correlação das dimensões do trato intestinal com o peso ou com o comprimento da carcaça, tomado entre as articulações das sétima e oitava vértebras torácicas e lombo-sacra.

\section{MATERIAL E MÉTODO}

Medimos o trato intestinal, desde o torus piloricus até o anus, de 40 bovinos de raças européias, 20 machos e 20 fêmeas, exibindo dentição definitiva completa, portanto, com no mínimo 5 anos de idade, provenientes de várias zonas de criação do Estado do Rio Grande do Sul e sacrificados no Matadouro Municipal de Alegrete.
Imediatamente após o abate, separávamos as peças em bloco, já sem o fígado, seccionando, em sentido transversal o abomaso e, então, incisávamos o mesentério ao longo da pequena curvatura, de forma a retificar o intestino, retirando ainda o epíplon e o pâncreas. A seguir, sempre nas cinco primeiras horas depois do sacrifício, dispunhamos o órgão em superfície plana e horizontal, cuidando para não estirá-lo e, tomávamos assim, as medidas dos diversos segmentos considerados, isto é, duodeno, jejuno e ileo, em conjunto, ceco e cólon mais reto, anotando também, informes relativos ao peso das carcaças e a distância compreendida entre as junturas da sétima e oitava vértebras torácicas e lombo-sacra, valores estes considerados como padrão, buscando possível dependência entre comprimento do tubo entérico e tamanho do animal.

As informações computadas foram submetidas estatisticamente à análise de correlação e ao teste de hipóteses de Student (test $t$ ).

\section{RESULTADOS}

Nas mensurações efetuadas, o comprimento médio total ou, das diferentes secções do intestino, quando levados em conta ambos os sexos e cada um dêles separadamente, alcançou, em metros, os valores discriminados na tabela abaixo:

\begin{tabular}{|c|c|c|c|c|c|}
\hline \multirow{2}{*}{\begin{tabular}{|} 
SEGMENTOS \\
UNTESTI \\
SEXOS NAIS
\end{tabular}} & \multirow{2}{*}{$\begin{array}{r}\text { INTESTINO } \\
\text { DELGADO }\end{array}$} & \multicolumn{3}{|c|}{ INTESTINO GROSSO } & \multirow{2}{*}{$\begin{array}{c}\text { COMPRIMENTO } \\
\text { TOTAL }\end{array}$} \\
\hline & & CECO & COLLON + RETO & TOTAL & \\
\hline MACHOS & $35,61 \pm 3,09$ & $0,57 \pm 0,06$ & $7,08 \pm 0,55$ & $7,65 \pm 0,64$ & $43,26 \pm 3,29$ \\
\hline FEMEAS & $36,21 \pm 2,21$ & $0,59 \pm 0,07$ & $7,08 \pm 0,51$ & $7,67 \pm 0,53$ & $43.88 \pm 2,53$ \\
\hline $\begin{array}{l}\text { MACHOS } E \\
F E M E A S\end{array}$ & $35,91 \pm 2,63$ & $0,58 \pm 0,06$ & $7,08 \pm 0,57$ & $7,66 \pm 0,59$ & $43,57 \pm 2,89$ \\
\hline
\end{tabular}

Cabe ainda acrescentar terem sido consignadas como medidas individuais respectivamente máxima e mínima, do intestino delgado - 43,49m e $28,53 \mathrm{~m}$; do ceco $-0,76 \mathrm{~m}$ e $0,46 \mathrm{~m}$; do cólon e reto $-9,49 \mathrm{~m}$ e $5,35 \mathrm{~m}$; do intestino grosso, em conjunto $-10,25 \mathrm{~m}$ e $5,87 \mathrm{~m}$; enfim, de todo o trato entérico $-53,74 \mathrm{~m}$ e $36,45 \mathrm{~m}$.
Quanto ao comprimento total do órgão ou de suas diversas porções, bem como o peso da carcaça e o espaço compreendido entre as junturas da sétima e oitava vértebras torácicas e lombo-sacra, assinaladas para cada animal, acham-se expostos nos Quadros I (machos) e II (fêmeas).

Finalmente, devemos aduzir que a aná- 
lise estatistica (test $\mathrm{t}$ ) realizada ao nivel de rejeição de $5 \%$ não revelou diferenças significantes ao confrontarmos os dados obtidos para os dois sexos; da mesma forma, não foi estabelecida correlação ao estudarmos possivel interdependência do comprimento total do intestino, quer com o peso da carcaça, quer com a aludida distância tomada sobre a coluna vertebral.

\section{COMENTẢRIOS}

Embora os AA. clássicos consultados (Tabela I) registrem a extensão de alguns segmentos intestinais ou de todo o trato em conjunto, não fornecem detalhes sobre a raça, o sexo, a idade, e mesmo, o número dos animais tomados para modelo nas aludidas descrições, tornando assim, difícil estabelecer confronto entre aquelas medidas e os dados aferidos em nossas mensuraçōes; o mesmo diga-se no tocante aos resultados de BLACK. SEMPLE e LUSH ${ }^{1}$, visto trabalharem estes AA. com bovinos jovens e, ao contrário, serem os nossos dados obtidos em animais adultos. Por sua vez, GIROLAMO ${ }^{9}$, em estudos sobre morfologia e dimensões do intestino de búfalos e bovinos, registra para 10 destes (3 machos e 2 fêmeas) medidas que, apesar de consignadas em espécimes de raças européias, fazem a possivel comparação com nossos resultados pois, o mencionado $\mathrm{A}$. considera, em grupo, individuos de diferentes idades, inclusive jovens, não atentando para o fato, mencionado por SCHMALTZ, de surpreender-se variações na extensão do trato intestinal, até o completo desenvolvimento do animal, cabendo esclarecer, fundamentarem-se nossos registros, ao contrário, na análise de amostra homogeneamente integrada por exemplares adultos.

Atentando agora aos valores médios, determinados por PAIVA e BORELLI ${ }^{19}$ para os diferentes segmentos do trato entérico em zebuínos, isto é, intestino delgado $31,15 \mathrm{~m}$, ceco $-\mathbf{0 , 5 1} \mathrm{m}$, cólon terminal e reto $-6,80 \mathrm{~m}$, intestina grosso em conjunto $\rightarrow$ $7,31 \mathrm{~m}$ e comprimento total do intestino $38,84 \mathrm{~m}$, podemos apurar face às dimensões médias por nós apuradas para taurinos, vale dizer, obedecida a mesma ordem de enumeração, $35,91 \mathrm{~m}, 0,58 \mathrm{~m}, 7,08 \mathrm{~m}, 7,66 \mathrm{~m}$ e
$43,57 \mathrm{~m}$, diferença estatisticamente significante no atinente ao comprimento da porção delgado e, por interferência desta, na extensão total do tubo intestinal, ressaltando, não terem sido verificadas divergências com significação estatística entre Bos taurus e Bos indicus no tocante ao comprimento, anotado para as diferentes seções do intestino grosso.

Por fim, cumpre acrescentar que o estudo estatistico não revelou diferenças significantes quando cotejados os valores médios obtidos para os sexos e, nem mesmo, correlação do comprimento total do intestino, seja com o peso da carcaça, seja com sua medida padrão, tomada desde a juntura das sétima e oitava vértebras torácicas até a lombo-sacra.

\section{CONCLUSÕES}

Com base nos valores assinalados nas mensurações de 40 tratos intestinais recolhidos de bovinos de raças européias, 20 machos e 20 fêmeas, com mais de 5 anos de idade, estabelecemos as conclusões abaixo discriminadas:

1 - 0 intestino delgado alcança, em média, $35,61 \mathrm{~m}$ nos machos e $36,21 \mathrm{~m}$ nas fêmeas, mostrando, então, média geral de $35,9] \mathrm{m}$, sendo a medida máxima registrada, $43,49 \mathrm{~m}$ e a mínima, $28,53 \mathrm{~m}$;

2 - O ceco apresenta o comprimento médio de $0,57 \mathrm{~m}$ nos machos e $0,59 \mathrm{~m}$ nas fêmeas, ou, $0,58 \mathrm{~m}$ como média geral, compreendida entre os extremos de 0,76 e $0,46 \mathrm{~m}$;

3 - $\mathrm{O}$ cólon e o reto, conjuntamente, exibem, em média, $7,08 \mathrm{~m}$, para ambos os sexos, coincidente, assim, com a média geral, tendo sido consignados valores limites de 9,49 e 5,35m;

4- $\mathrm{O}$ intestino grosso, portanto, atinge a extensão média de $7,65 \mathrm{~m}$, nos machos e $7,67 \mathrm{~m}$ nas fêmeas, sendo $7,66 \mathrm{~m}$ a média geral e a maior e a menor medidas, respectivamente, $10,25 \mathrm{~m}$ e $5,87 \mathrm{~m}$;

5 - Finalmente, para o trato intestinal, considerado no seu todo, encontramos, em 
média, $43,26 \mathrm{~m}$ nos machos e $43,88 \mathrm{~m}$, nas fêmeas, ou seja, média geral de $43,57 \mathrm{~m}$ e comprimentos máximo de $53,74 \mathrm{~m}$, e minimo de $36,45 \mathrm{~m}$;

6 - A análise estatística não revela diferenças significantes quando confrontados os valores médios aferidos para machos e fêmeas, bem como, não estabelece correlação da extensão total do intestino, ou com o peso da carcaça ou, com seu comprimento;
7 - Comparando nossos resultados com os assinalados para animais de origem indiana, apuramos a existência de diferença estatisticamente significante no relativo ao intestino delgado e, por interferência deste, do comprimento total do trato intestinal, visto não computarmos divergências dotadas de significância, no tocante a diferentes porções do intestino grosso.

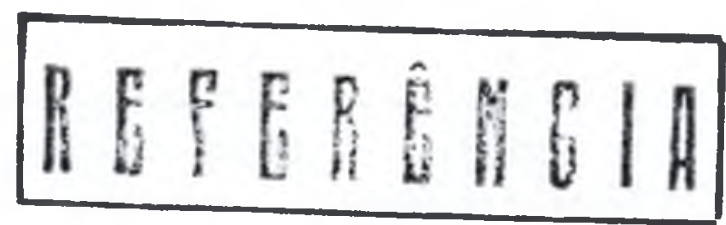

Rev. Fac. Med. vet. Zootec. Univ. S. Paulo, 14(2):189-197, 1977. 


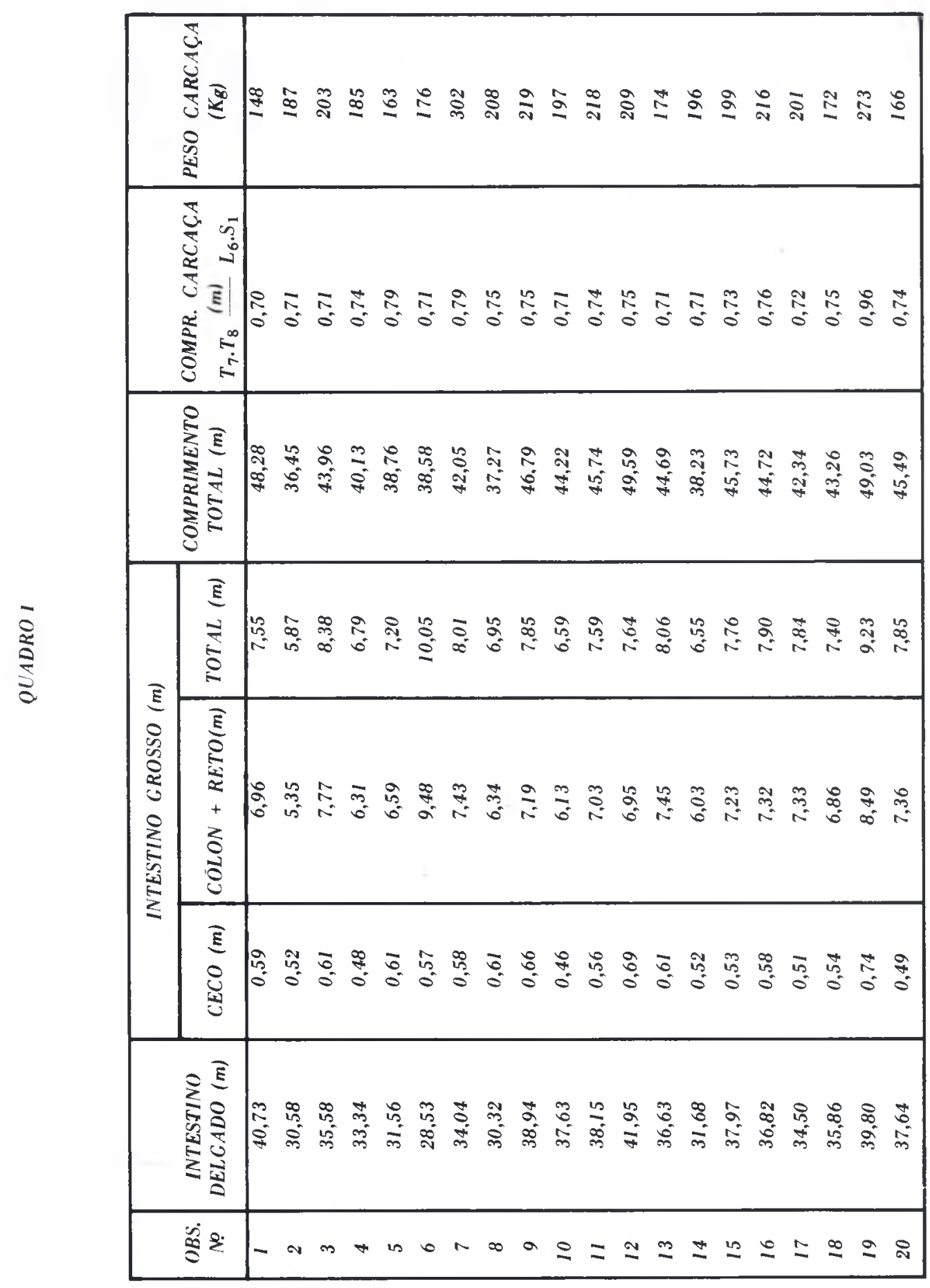




\begin{tabular}{|c|c|c|}
\hline & 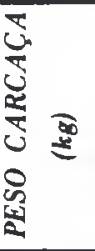 & 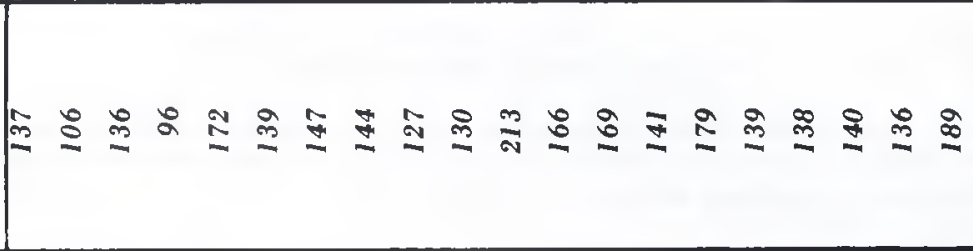 \\
\hline & 焉 & 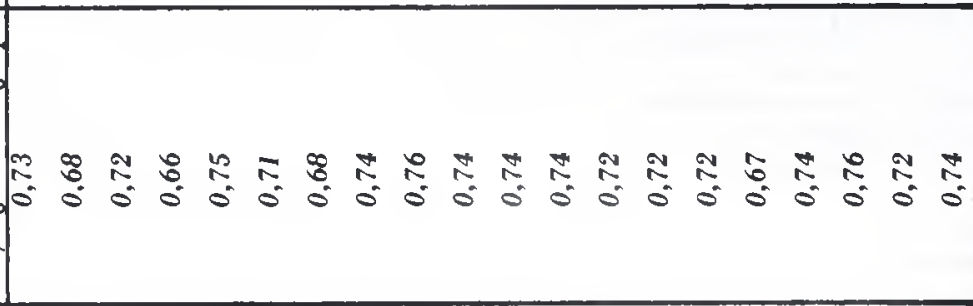 \\
\hline & 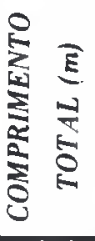 & 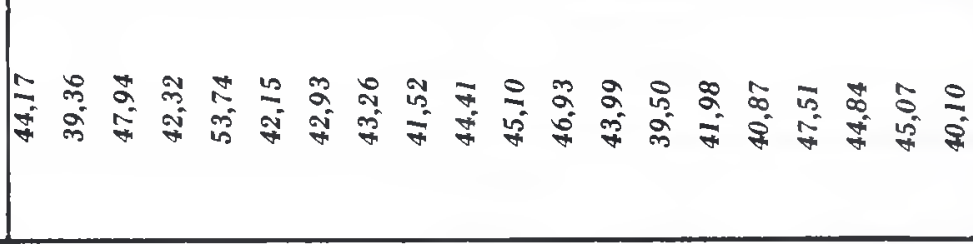 \\
\hline \multirow{3}{*}{ 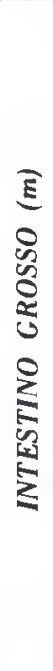 } & 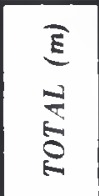 & 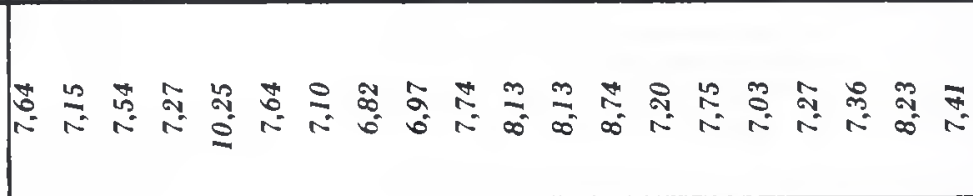 \\
\hline & 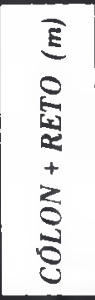 & 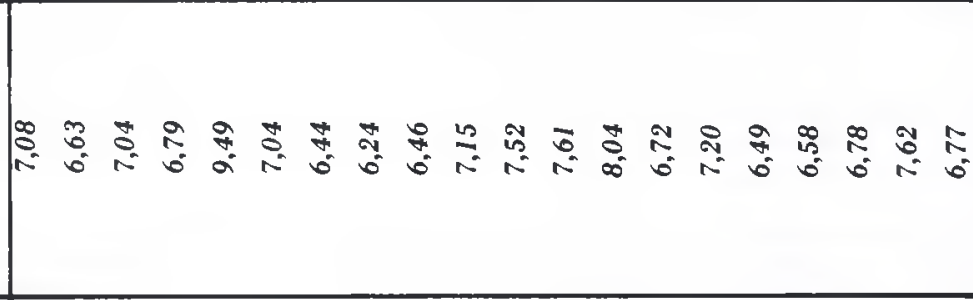 \\
\hline & $\begin{array}{l}\text { E } \\
0 \\
\text { 跑 }\end{array}$ & 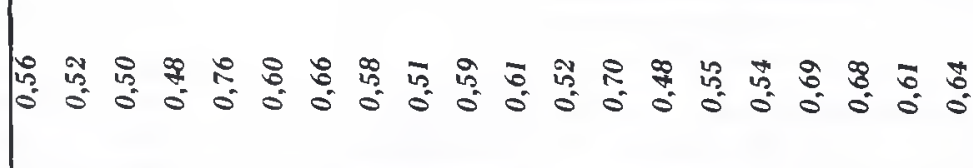 \\
\hline & 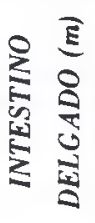 & 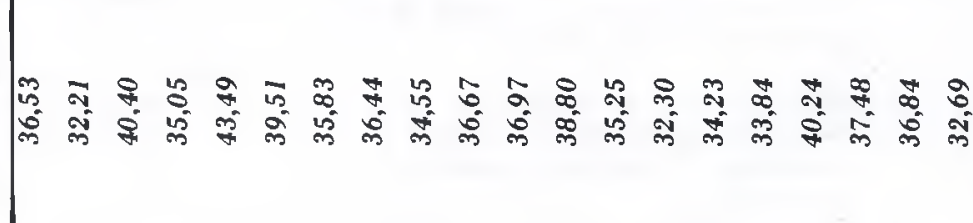 \\
\hline & $\dot{0}$ & 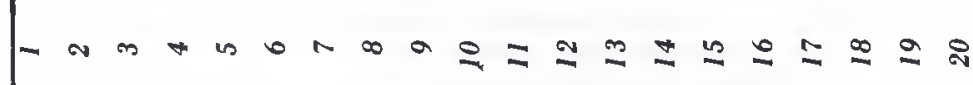 \\
\hline
\end{tabular}


RFMV-A/21

PAIVA, O.M.; BORELLI, V.; PEDUTI NETO. J. The lenght of the intestine in bovines. Rev. Fac. Med. vet. Zootec. Univ. S. Paulo, 14(2):189-197, 1977.

SUMMARY: The AA. studied the lenght of the various enteric tracts as well as their full length in 40 adult bovines of European origin ( 20 males and 20 females) and achieved the following average, once there was no significant difference concerning sex:

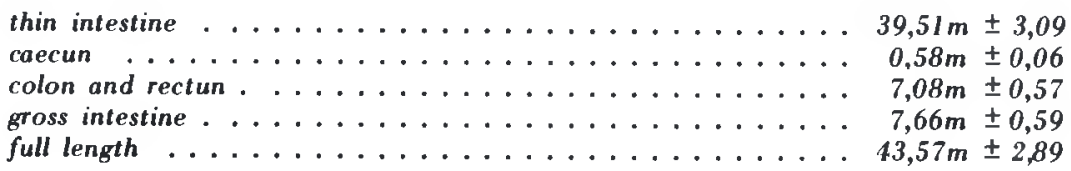

On the other hand, there was no correlation between the full length of the intestine and the weight or length of the carcasses, nevertheless significant differences bet ween the average obtained in Bos taurus and Bos indicus concerning the length of the thin intestine and the full length have been verified.

UNITERMS: Anatomy, bovine *; Intestine, anatomy.

\section{REFERÊNCIAS BIBLIOGRĀFICAS}

1 - BLACK, W.H.; SEMPLE, A.T.; LUSH, J.L. Beef production and quality as influenced by crossing Brahman with Hereford and Shorthorn cattle. Tech. Bull.U.S. Dep. Agric., (417): 1934.

2- BRUNI, A.C. \& ZIMMERL, U. Anatomia degli animali domestici. Milano, Francesco Vallardi, 1949, v.2.

3 - CARADONNA,G.B. \& IN ZIMMERL, U. Trattato di anatomia veterinaria. Milano, Francesco Vallardi, 1930, v.2.

4 - CARADONNA, G.B.; IN BOSSI, V.; CARADONNA, G.B.; SPAMPANI, G.; VARALDI, L.; ZIMMERL, U. Trattato di anatomia veterinaria. Milano, Francesco Vallardi, s.d. v.2.

5 - DOBBERSTEIN, J.; KOCH, T.; IN PIEKOS, M.; PILARSKI, W.; ROSKOSZ, T. Obserwacje nad dtugoscia jelita e zubra-Bison bonasus L. Folia Morphologica, 9: 69-79, 1958.

6 - ELLENBERGER, W. \& BAUM, H. Handbuch der vergleichenden Anatomie der Hanstiere. 17ed. Berlin, Julius Springer, 1932.

7 - FAVILLI, N. Nozioni comparate di anatomia e fisiologia degii animali agricoli. Milano, Francesco Vallardi, 1943.

8 - FAVILLI, N. Nozioni comparate di anatomia e fisiologia degli animali rura- li. Torino, Unione Tipografico - Editrice Torinesse, 1931 .

9 - GIROLAMO, A. Rilievi comparativi sulla morfologia e sulla lunghezza dell'intestino fra. Bos taurus e Bos bubalus Neove Vet., 1: 1-6, 1950.

10 - GONZALES Y GARCIA, J. \& ALVAREZ, R.G. Anatomia comparada de los animales domesticos. 7.ed. Madrid, Gráficas Canales, 1961.

11 - KATO, K. Katiku hikaku Kaibon zusitsu. /Exposição gráfica da anatomia comparada dos animais domésticos./ 6.ed. Tokio, Yokendo, 1963. v.1.

12 - KLIMOW, A.; In PEKOS, M.; PILARSKI, W.; ROSKOSZ, T. Obserwacje nad dtugoscia jelita u zubra - Bison bonasus L. Folia Morphologica, 9: 69-79, 1958.

13 - LEPOUTRE, L. Notes du cours d'anatomie comparée des animaux domestiques. Gembloux, J. Duculot, 1921.

14 - LESBRE, F.X. Précis d'anatomie comparée des animaux domestiques. Paris, J.B. Baillière et fils, 1922. v.1.

15 - MARTIN, P. \& SCHAUDER, W. Lehrbuch der Anatomie der Hanstiere. 3.Auf. Stuttgart, Schickhardt \& Ebner, 1935, v.3. t.2. 
16 - MASSUI, K. Katiku hikaku Kaibon gaku. /Anatomia comparada dos animais do mésticos./ 7.ed. Tokio, Yokendo, 1960.

17 - MONGIARDINO, T. \& In GIROLAMO A. Rilievi comparativi sulla morfologia e sulla lunghezza dell'intestino fra Bos Taurus e Bos bubalus. Nuova Vet., 1 : $1-6,1950$

18 - MONTANÉ, L. \& BOURDELlE, E. Anatomie régionale des animaux domestiques. Paris, J.B. Bailliére, 1917, v.2.

19 - PAIVA, O.M. \& BORELli, V. /Comprimento total do intestino de bovinos azebuzados. São Paulo, 1962. . Trabalho apresentado à XVIII. Conferência Anual da Sociedade Paulista Medicina Veterinária./

20 - SCHMALTZ, R.; In PIEKOS, M.; PILARSKI, W.; ROSKOSZ, T. Obserwage nad dtugoscia jelita u zubra - Bison bonasus L. Folia Morphologica, 9: 69-79, 1958.

21 - SCHUMMER, A.; NICKEL, R.; In NICKEL, R.; SCHUMMER, A.; SEIFERLR, E, Lchrbuch der Anatomie der Haustiere. Berlin, Paul Parey, 1960. v.2.

22 - SCHWARZE, E. \& SCHRODER, L. Kompendium der Veterinar - Anatomie. Jena, Gustav Fischer, 1962, v.2.

23 - SISSON, S. \& GROSSMANN, J.D. Anatomia de los animales domesticos. Barcelona, Salvat Editores, 1965.

24 - ZIMMERL, U. Anatomia topográfica veterinária. Milano, Francesco Vallardi, 1949. v.2.

Recebido para publicação em 3-8-77 Aprovado para publicação em 30-8-77 